\title{
Characterization of serious adverse drug reactions as cause of emergency department visit in children: a 5-years active pharmacovigilance study
}

Niccolò Lombardi ${ }^{1 *}$, Giada Crescioli ${ }^{1+}$, Alessandra Bettiol ${ }^{1}$, Ettore Marconi ${ }^{1}$, Antonio Vitiello ${ }^{1}$, Roberto Bonaiuti ${ }^{1}$, Anna Maria Calvani ${ }^{2}$, Stefano Masi ${ }^{3}$, Ersilia Lucenteforte ${ }^{1}$, Alessandro Mugelli ${ }^{1}$, Lisa Giovannelli ${ }^{1}$ and Alfredo Vannacci ${ }^{1}$

\begin{abstract}
Background: To describe frequency, preventability and seriousness of adverse drug reactions (ADRs) in children as cause of emergency department (ED) admission and to evaluate the association between specific factors and the reporting of ADRs.

Methods: A retrospective analysis based on reports of suspected ADRs collected between January 1st, 2012 and December 31st, 2016 in the ED of Meyer Children's Hospital (Italy). Demographics, clinical status, suspected drugs, ADR description, and its degree of seriousness were collected. Logistic regression was used to estimate the reporting odds ratios (RORs) with $95 \%$ confidence intervals (Cls) of potential predictors of ADR seriousness.

Results: Within 5 years, we observed 834 ADRs (1100 drug-ADR pairs), of whom 239 were serious; of them, 224 led to hospitalization. Patients were mostly treated with one drug. Among patients treated with more than one drug, 78 ADRs presented a potential interaction. The most frequently reported ADRs involved gastrointestinal system. The most frequently reported medication class was antinfectives. Risk of serious ADR was significantly lower in children and infants compared to adolescents (ROR 0.41 [95\% Cl: 0.27-0.61] and 0.47 [0.32-0.71], respectively), and it was significantly increased in subjects exposed to more than one drug (ROR 1.87 [1.33-2.62] and 3.01 [2.07-4.37] for subjects exposed to 2 and 3 or more drugs, respectively). Gender, interactions and off-label drug use did not influence the risk of serious ADRs.
\end{abstract}

Conclusion: Active surveillance in pharmacovigilance might represent the best strategy to estimate and characterize the clinical burden of ADRs in children.

Keywords: Pediatrics, Adverse events, Safety, Observational study, Preventability, Drug interactions

\section{Background}

Adverse drug reactions (ADRs) are the most common cause of iatrogenic harm in health care and have recently received attention in national patient safety initiatives [1]. Moreover, ADRs are a significant cause of emergency department (ED) visits [2].

\footnotetext{
*Correspondence: niccolo.lombardi@unifi.it

${ }^{\dagger}$ Equal contributors

'Department of Neurosciences, Psychology, Drug Research and Child Health, Section of Pharmacology and Toxicology, University of Florence, viale G. Pieraccini, 6, 50139 Florence, Italy

Full list of author information is available at the end of the article
}

Most studies on hospital admissions related to medication use focus on adult patients [3-8]. Fortunately, the burden of ADR-related ED admissions in children seems to be lower than observed in adults $(5 \%-25 \%$ of all ED admissions) [3, 9], ranging from $0.4 \%$ to $10.3 \%$ of all children with a pooled estimate of $2.9 \%$ [10].

In this context, risk factors for ADRs in children are still poorly characterized. In fact, pharmacotherapy in children differs from adults, thus the spectrum of ADRs in children may differ as well [11], and certain subgroups are at higher risk, such as children exposed to cancer chemotherapy [12]. Furthermore, children are more

(C) The Author(s). 2018 Open Access This article is distributed under the terms of the Creative Commons Attribution 4.0 International License (http://creativecommons.org/licenses/by/4.0/), which permits unrestricted use, distribution, and 
often exposed to off-label medications, that potentially represent a greater risk for ADRs [13]. Because part of these medication-related events may be preventable [14], more knowledge on occurrence and content of ADRs in pediatric setting is necessary, with efforts focused on reducing medication errors and inappropriate prescribing [1].

EDs are an essential part of health care systems, serving as an interface between hospitals and communities, and could constitute the most important source of information about the frequency, seriousness, and economic burden of ADRs $[15,16]$.

Preventing ADRs and their related ED admissions in outpatients remains a public health and a patient safety challenge. Aims of this study are to describe frequency, preventability and seriousness of ADRs in children, and to evaluate the association between specific factors and the reporting of ADRs, by means of a 5-years long active pharmacovigilance study in ED.

\section{Methods}

This is a retrospective study performed on reports of suspected ADRs collected between January 1st, 2012 and December 31st, 2016 in the ED of Anna Meyer Children's University Hospital of Florence (Italy), as part of the study "Epidemiological Monitoring of Adverse Drug Reactions in Emergency Department" (MEREAFaPS), an on-going multicenter Italian active pharmacovigilance project. We analyzed all suspected ADRs reported from patients having at least one drug- or vaccine-related event.

All suspected ADRs leading to ED admission were identified from ED clinical charts and hospitalization data from the hospital discharge database. For each ADR report, we recorded demographic characteristics (age, gender, ethnic group), and patient clinical status on ED admission. Anatomical Therapeutic Chemical (ATC) classification system was used to code ongoing therapy (suspect and concomitant medications, administration route, therapy duration, and dosages). We also recorded all therapeutic indications of suspected drugs. The description of the ADR according to diagnosis and symptoms, was coded using the Medical Dictionary for Regulatory Activities (MedDRA) and organized by System Organ Class (SOC) [17]. ADR seriousness was classified according to the World Health Organization (WHO) criteria [17] as fatal, life-threatening, or requiring hospitalization of the patient, or causing serious/permanent disability.

The primary outcome was the frequency of total and serious ADRs leading to ED admission. The most frequently reported SOC and ATC classes were also evaluated. For each drug-ADR pair, causality and preventability (categorized as definitely or probably preventable, or not preventable) were assessed using the Naranjo [18] scale and Schumock and Thornton [19] algorithm, respectively.
For the causality assessment of vaccine-related ADRs, the specific WHO [20, 21] algorithm was used. Data on medications were also analysed for drug-drug interactions (DDIs) by using the drug interaction software Micromedex Drug-REAX System (Thomson Reuters Healthcare Inc., Greenwood Village, Colorado, United States), available online with restricted access [22, 23].

The specific factors associated with the reporting of ADRs, such as age classes, sex, number of suspected drugs, DDIs and on-label vs off-label use were also analysed. The off-label use in children was defined as the use of a drug already covered by a Marketing Authorisation, in an unapproved way in terms of therapeutic indication, posology, formulation and route of administration [24].

Descriptive statistics was used to summarize data. Categorical data were reported as frequencies and percentages, whereas continuous data were reported as means with standard errors. Univariate and multivariate logistic regression were used to estimate the reporting odds ratios (RORs) with 95\% confidence intervals (CIs) of potential predictors of ADR seriousness. ROR values for each variable were reported both as crude values and adjusted for all the other considered variables. All results were considered to be statistically significant at $p<0.05$. Data management and statistical analysis were carried out using STATA 14.

Tuscany approved the present study with the Notification number 1225 - December 21, 2009. The local institutional ethics committee of the coordinating center (Comitato Etico di Area Vasta Nord "CEAVNO" per la Sperimentazione Clinica, Azienda Ospedaliero-Universitaria Pisana) approved the study according to the legal requirements concerning observational studies (Study number 3055/ 2010, Protocol number 45288 - August 6, 2014).

\section{Results}

During the 5-year study period, a total of 221,528 ED admissions was evaluated; of them, 492 were ADRrelated, with an overall rate of 2.2 per 1000 ED admissions. Table 1 shows data of pediatric patients who experienced an ADR-related ED admission. We observed 834 ADRs, of whom 239 were serious (28.66\%) and, of them, 224 led to hospitalization. No ADR led to death. Most of ADRs occurred in Caucasians (90.65\%), with a mean patients' age ( \pm standard error) of $62.53 \pm 2.09$ months. The male/female rate of ADRs was 1.17, i.e. comparable to the all-cause ED admission rate observed in Meyer Children's University Hospital. A total of 483 ADRs (57. 91\%) had an improvement and 48 (5.76\%) had a complete resolution. For 285 ADRs (34.17\%) data on the outcome were not available. Overall, patients were mostly treated with only one drug at time of ADR occurrence. Among patients treated with two or more drugs, 78 ADRs (20.21\%) presented a potential DDI, which was 
Table 1 Characteristics of pediatric patients admitted to ED for ADR

\begin{tabular}{|c|c|}
\hline & Tot ADRs N (\% out of 834) \\
\hline $\mathrm{N}$ involved patients & 492 \\
\hline \multicolumn{2}{|l|}{ N ADRs/patients } \\
\hline 1 & $256(52.03)$ \\
\hline 2 & $155(31.50)$ \\
\hline $3+$ & $81(16.46)$ \\
\hline \multicolumn{2}{|l|}{ Age } \\
\hline Mean \pm standard error, months & $62.53 \pm 2.09$ \\
\hline \multicolumn{2}{|l|}{ Age classes } \\
\hline New-borns (<1 month) & $7(0.84)$ \\
\hline Infants (1-23 months) & $332(39.81)$ \\
\hline Children (24-143 months) & $368(44.12)$ \\
\hline Adolescents (144-192 months) & $127(15.23)$ \\
\hline \multicolumn{2}{|l|}{ Sex } \\
\hline Male & $449(53.48)$ \\
\hline Female & $385(46.16)$ \\
\hline \multicolumn{2}{|l|}{ Ethnic group } \\
\hline Caucasian & $756(90.65)$ \\
\hline Others & $25(3.00)$ \\
\hline Not available & $53(6.35)$ \\
\hline \multicolumn{2}{|l|}{ Tot number of drugs } \\
\hline 1 & $448(53.72)$ \\
\hline 2 & $255(30.58)$ \\
\hline $3+$ & $131(15.71)$ \\
\hline \multicolumn{2}{|l|}{ Seriousness } \\
\hline Hospitalization & $224(26.86)$ \\
\hline Congenital abnormalities/ Deficit & $1(0.12)$ \\
\hline Other clinically relevant conditions & $14(1.68)$ \\
\hline \multicolumn{2}{|l|}{ Outcome } \\
\hline Complete resolution & $48(5.76)$ \\
\hline Improvement & $483(57.91)$ \\
\hline Invariant situation/worsening & $18(2.16)$ \\
\hline Not available & $285(34.17)$ \\
\hline \multicolumn{2}{|l|}{ Drug-drug interactions ${ }^{a}$} \\
\hline No & $308(79.79)$ \\
\hline Yes & $78(20.21)$ \\
\hline Mild & $4(1.04)$ \\
\hline Moderate & $17(4.40)$ \\
\hline \multirow[t]{2}{*}{ Severe } & $57(14.77)$ \\
\hline & $\begin{array}{l}\text { Tot drug ADR-pairs N } \\
\text { (\% out of } 1100)\end{array}$ \\
\hline Drugs (vaccines excluded) & $711(64.64)$ \\
\hline \multicolumn{2}{|l|}{ Causality } \\
\hline In doubt & 18 \\
\hline Possible & 301 \\
\hline
\end{tabular}

Table 1 Characteristics of pediatric patients admitted to ED for ADR (Continued)

\begin{tabular}{ll}
\hline & Tot ADRs N (\% out of 834) \\
\hline Probable & 310 \\
Certain & 82 \\
Vaccines & $389(35.36)$ \\
Causality & \\
Not associable & 6 \\
Undefined & 50 \\
Associable & 333
\end{tabular}

${ }^{\mathrm{a}}$ Percentage calculated on the total number of ADRs occurred in patients with more than one drug

severe in 57 cases $(14.77 \%)$. The total number of drugADR pairs was 1100 . Of them, 711 drug-ADR pairs were related to non-vaccines drugs; considering causality assessment, 82 were certain, 310 probable, 301 possible and 18 in doubt. On the other hand, 389 (35.36\%) were related to vaccines; in particular, considering the evaluation of causality assessment, 333 were considered as associable, 50 as undefined and 6 were not associable.

Table 2 reported the distribution of ADRs according to SOC classification. The most frequently reported SOCs were: gastrointestinal disorders (27.34\%), followed by general disorders and administration site conditions (21.46\%), skin and subcutaneous tissue disorders (16. $19 \%)$, nervous system disorders (12.95\%) and metabolism and nutrition disorders (3.60\%). The majority of ADRs for each SOC were non-serious.

The drug-ADR pairs (stratified by ATC classes, level I) most commonly involved in ED admissions, overall and stratified according to ADRs seriousness, preventability and off-label use are listed in Table 3. Out of 1100 drugADR pairs, 711 were not related to vaccines; of them more than $30 \%$ were serious, $24 \%$ preventable and $8.7 \%$ related to off-label use. The most frequently reported ATC class was antinfectives for systemic use (ATC class J, excluded $\left.\mathrm{J}^{*}{ }^{*}\right)$ accounting for 288 drug-ADR pairs (40. $51 \%)$. Of them, $23.96 \%$ were serious, $24.19 \%$ were preventable and $0.69 \%$ were related to off-label use. The second most frequently reported ATC class was nervous system medication (ATC class N), accounting for 168 drug-ADR pairs (23.63\%), of whom 48.21, 48.81 and 10. $71 \%$ were serious, preventable and related to off-label use, respectively. All other ATC classes accounted for 8 . 30 to $0.56 \%$ of drug-ADR pairs.

The most frequent suspected active principles (APs) among antinfectives for systemic use agents and nervous system medications are reported in Additional file 1: Table S1.

Out of 1100 drug-ADR pairs, 389 were related to vaccines (35.36\%); of them, $28 \%$ and $6.9 \%$ were serious and preventable, respectively (Table 3 ). The most 
Table 2 Distribution of ADRs according to SOC classification

\begin{tabular}{|c|c|c|}
\hline & Tot ADRs N (\% out of 834) & Serious ADRs N (\% out of corresponding SOC) \\
\hline \multicolumn{3}{|l|}{ SOC } \\
\hline Gastrointestinal disorders & $228(27.34)$ & $68(29.82)$ \\
\hline General disorders and administration site conditions & $179(21.46)$ & $41(23.56)$ \\
\hline Skin and subcutaneous tissue disorders & $135(16.19)$ & $17(12.59)$ \\
\hline Nervous system disorders & $108(12.95)$ & $45(41.67)$ \\
\hline Metabolism and nutrition disorders & $30(3.60)$ & $11(36.67)$ \\
\hline Respiratory, thoracic and mediastinal disorders & $27(3.24)$ & $8(29.63)$ \\
\hline Musculoskeletal and connective tissue disorders & $27(3.24)$ & $11(40.74)$ \\
\hline Psychiatric disorders & $18(2.16)$ & $7(38.89)$ \\
\hline Eye disorders & $13(1.56)$ & $5(38.46)$ \\
\hline Cardiac disorders & $12(1.44)$ & $5(41.67)$ \\
\hline Ear and labyrinth disorders & $10(1.20)$ & $1(10)$ \\
\hline Investigations & $8(0.96)$ & $3(37.50)$ \\
\hline Infections and infestations & $8(0.96)$ & $1(12.50)$ \\
\hline Vascular disorders & $7(0.84)$ & $2(28.57)$ \\
\hline Injury, poisoning and procedural complications & $7(0.84)$ & $4(57.14)$ \\
\hline Blood and lymphatic system disorders & $5(0.60)$ & $3(60.00)$ \\
\hline Reproductive system and breast disorders & $4(0.48)$ & $0(0.00)$ \\
\hline Renal and urinary disorders & $4(0.48)$ & $3(75.00)$ \\
\hline Immune system disorders & $3(0.36)$ & $3(100.00)$ \\
\hline Surgical and medical procedures & $1(0.12)$ & $1(100.00)$ \\
\hline
\end{tabular}

Table 3 Distribution of drug-ADR pairs according to ATC classification (level I), overall and stratified according to seriousness, preventability and off-label use

\begin{tabular}{|c|c|c|c|c|}
\hline & $\begin{array}{l}\text { Tot drug-ADR pairs } \\
N(\% \text { out of } 1100)\end{array}$ & $\begin{array}{l}\text { Seriousness } \\
\mathrm{N}(\% \text { out of ATC level I) }\end{array}$ & $\begin{array}{l}\text { Preventability } \\
\text { N (\% out of ATC level I) }\end{array}$ & $\begin{array}{l}\text { Off-label use } \\
N \text { (\% out of ATC level I) }\end{array}$ \\
\hline Related to Vaccines & $389(35.36)$ & $109(28.02)$ & $27(6.94)$ & - \\
\hline Not related to Vaccines (ATC level I) & $711(64.64)$ & $226(31.79)$ & $172(24.19)$ & $62(8.72)$ \\
\hline $\begin{array}{l}\text { J - Antinfectives for systemic use } \\
\text { (excluded vaccines - J07) }\end{array}$ & $288(40.51)$ & $69(23.96)$ & $15(5.21)$ & $2(0.69)$ \\
\hline N - Nervous system & $168(23.63)$ & $81(48.21)$ & $82(48.81)$ & $18(10.71)$ \\
\hline A - Alimentary tract and metabolism & $59(8.30)$ & $16(27.12)$ & $25(42.37)$ & $3(5.08)$ \\
\hline M - Musculoskeletal system & $53(7.45)$ & 18 (33.96) & 9 (16.98) & $0(0.00)$ \\
\hline C - Cardiovascular system & $39(5.49)$ & $17(43.59)$ & $22(56.41)$ & $18(46.15)$ \\
\hline H - Systemic hormonal preparations & $31(4.36)$ & $7(22.58)$ & $6(19.35)$ & $5(16.13)$ \\
\hline $\mathrm{R}$ - Respiratory system & $27(3.80)$ & $3(11.11)$ & $2(7.41)$ & $3(11.11)$ \\
\hline L - Antineoplastic and immunomodulating agents & $13(1.83)$ & $4(30.77)$ & $2(15.38)$ & $2(15.38)$ \\
\hline B- Blood and blood-forming organs & $11(1.55)$ & $6(54.55)$ & $3(27.27)$ & $5(45.45)$ \\
\hline D - Dermatologicals & $10(1.41)$ & $3(30.00)$ & $1(10.00)$ & $0(0.00)$ \\
\hline G - Genitourinary system and sex hormones & $4(0.56)$ & $2(50.00)$ & $4(100.00)$ & $4(100.00)$ \\
\hline P - Antiparasitic products & $4(0.56)$ & $0(0.00)$ & $0(0.00)$ & $0(0.00)$ \\
\hline S - Sensory organs & $4(0.56)$ & $0(0.00)$ & $1(25.00)$ & $2(50.00)$ \\
\hline
\end{tabular}


frequent suspected vaccines were Meningococcus B, multicomponent vaccine $(n=67$, of whom $32.84 \%$ serious), Diphtheria-haemophilus influenzae B-pertussispoliomyelitis-tetanus-hepatitis B $(n=57$, of whom 45 . $61 \%$ serious), and Pneumococcus, purified polysaccharides antigen conjugated $(n=55$, of whom $32.73 \%$ serious) (Additional file 2: Table S2).

Table 4 reports the distribution of serious and nonserious drug-ADR pairs and the potential associations between serious ADR risk and age, gender, total number of pharmacological treatments and presence of DDIs. On-label and off-label use and relative associations were also evaluated. Seriousness of ADRs significantly differed among age classes and according to the total number of drugs administered $(p<0.001)$. Risk of having an ADR reported as serious was significantly lower in children and infants compared to adolescents (adjusted ROR of 0 . 41 [95\% CI: 0.27-0.61] and 0.47 [0.32-0.71], respectively). Risk of serious ADRs was significantly increased in subjects exposed to more than one drug (adjusted ROR of 1.87 [1.33-2.62] and 3.01 [2.07-4.37] for subjects exposed to 2 and 3 or more drugs, respectively) compared to subjects exposed to only one drug. On the other hand, the gender, the presence of DDIs and the on-label or off-label drug use did not influence the risk of having an ADR reported as serious.

\section{Discussion}

This study aimed to explore the frequency, preventability and seriousness of ADRs observed over a 5-year period in a single pediatric center in Italy, and to our knowledge it is the first study focused on serious ADRs with the aim of addressing the factors associated with the reporting of this kind of ADRs, in an Italian clinical pediatric setting.

In the present analysis, the rate of ADR-related ED admissions was 2.2 per 1000 admissions, notably lower than what is generally reported in literature $(0.4 \%$ to $10.3 \%$ of all children, pooled estimate of $2.9 \%$ ) [10]. In fact in a large systematic review [10], the rates of ADRs causing hospital admission ranged from $0.4 \%$ to $10.3 \%$ of all children (pooled estimate of $2.9 \%(2.6 \%, 3.1 \%))$. This can be explained by the fact that we calculated the actual rate of ADRs on all ED admissions, including also those not related to drug utilization. These findings are in keeping with what was reported in another Italian study [25] that showed similar results in terms of ATC classes and SOCs most frequently associated with ADRs in children. According to Rosafio et al. (2017) [25], the medication classes most frequently implicated were anti-infective drugs for systemic use (28.9\%) and central nervous system agents $(22.3 \%)$. The three most common symptom manifestations were dermatologic conditions (46.1\%), general

Table 4 Distribution of serious and non-serious drug-ADR pairs, and association between serious ADR risk and different factors, expressed as Reporting Odds Ratio (ROR)

\begin{tabular}{|c|c|c|c|c|c|}
\hline & $\begin{array}{l}\text { Non-serious drug-ADR pairs } \\
\text { N (\% out of } 765 \text { ) }\end{array}$ & $\begin{array}{l}\text { Serious drug-ADRs pairs } \\
N(\% \text { out of } 335)\end{array}$ & $p$-value & $\begin{array}{l}\text { Adjusted ROR of serious ADR } \\
{[95 \% \mathrm{Cl}]}\end{array}$ & $p$-value \\
\hline \multicolumn{6}{|l|}{ Age classes (FDA classification) } \\
\hline Adolescents (144-192 months) & $86(11.24)$ & $72(21.49)$ & $<0.001$ & Ref & \\
\hline Children (24-143 months) & 335 (43.79) & $123(36.72)$ & & $0.41[0.27-0.61]$ & $<0.001$ \\
\hline Infants (1-23 months) & $336(43.92)$ & $137(40.90)$ & & $0.47[0.32-0.71]$ & 0.001 \\
\hline New-borns (<1 month) & $8(1.05)$ & $3(0.90)$ & & $0.42[0.10-1.70]$ & 0.224 \\
\hline \multicolumn{6}{|l|}{ Sex } \\
\hline Male & $353(46.14)$ & $144(42.99)$ & 0.333 & Ref. & \\
\hline Female & $412(53.86)$ & $191(57.01)$ & & $0.77[0.59-1.02]$ & 0.072 \\
\hline \multicolumn{6}{|l|}{ Tot number of drugs } \\
\hline 1 & $347(45.36)$ & $101(30.15)$ & $<0.001$ & Ref. & \\
\hline 2 & $276(36.08)$ & $128(38.21)$ & & $1.87[1.33-2.62]$ & $<0.001$ \\
\hline $3+$ & $142(18.56)$ & $106(31.64)$ & & $3.01[2.07-4.37]$ & $<0.001$ \\
\hline \multicolumn{6}{|l|}{ Drug-drug interactions } \\
\hline No & $268(64.11)$ & $165(70.51)$ & 0.097 & & \\
\hline Yes & $150(35.89)$ & $69(29.49)$ & & $0.77[0.54-1.11]$ & 0.166 \\
\hline \multicolumn{6}{|l|}{ Off-label vs on-label use ${ }^{a}$} \\
\hline On-Label & $447(92.36)$ & $201(88.94)$ & 0.133 & Ref. & \\
\hline Off-label & $37(7.64)$ & $25(11.06)$ & & $1.56[0.90-2.73]$ & 0.115 \\
\hline
\end{tabular}

Adjusted ROR values are shown along with the respective $95 \%$ Confidence Intervals

${ }^{a}$ Calculated only on non-vaccine medications 
disorder and administration site conditions (29.7\%) and gastrointestinal symptoms (16.0\%).

Among the ADR reports collected in our study, "Antinfectives for systemic use" were the most common drugs, in agreement with a major national ADRs overview conducted in Italy in the last decade $[16,26]$. In particular, amoxicillin and amoxicillin/clavulanate were the most frequently involved APs. This evidence could be attributable to the fact that in Tuscany amoxicillin and amoxicillin/clavulanate are generally the most prescribed antibiotics in the pediatric population (14.06 and 23.08 defined daily dose/1000 inhabitants per day, respectively) [27]. The most reported SOC in the present study was "Gastrointestinal disorders", whereas in literature "Skin and subcutaneous tissue disorders" is generally the first SOC to be reported in this population subset [28]. This discrepancy could be related to the fact that dermatological ADRs are generally less serious than gastrointestinal ones, and therefore could be mainly managed by out-of-hospital healthcare professionals rather that in EDs.

Regarding vaccine-related ED admission, this study showed a higher number of drug-ADR pairs for "Meningococcus $\mathrm{B}$ multicomponent vaccine" than reported by Rosafio et al. [25]. This data are not surprising given the peculiar Tuscan scenery of meningococcal B disease high incidence and the correlated high vaccination rate in children in the past few years [29]. Anyway, in our study the most common ADRs related to vaccines were fever, injection-site hypersensitivity and edema, and local vasodilatation (data not shown), that cannot be considered life-threatening, although they led to an ED admission. Thus, considering the high number of vaccine doses administered in Tuscany every year [30], the total number of vaccines-related ADRs detected in the present study is reasonably low and provides a general perception of safety of vaccination in children [31].

The rate of serious ADRs compared to non-serious ones, was lower than observed in other studies, performed in both general and pediatric populations [17, 28]. The lower rate of serious ADRs in this sample may indicate a better quality and safety of prescribing in the setting evaluated. Among the drug classes that deserve particular attention in terms of preventable ADRs, there are "Antinfectives for systemic use" (ATC class J) and "Nervous system medications" (ATC class N). The clinical evaluation of the collected ADR reports identified several cases of misuse and abuse due to accidental drug ingestion for class N. Unintentional exposure among children is an important public health problem [32]. One out of 180 two-year-old child visits an ED for a medication poisoning each year [33], and, during 2010-2011, an average of 1499 children/year aged less than 6 years were evaluated in ED in the United States for unintentional exposure to drugs, including agents of ATC class $\mathrm{N}$ [34]. In this context, our evidences underline the importance of careful drug management by parents and caregivers [35], with the final goal of improving the strategies to prevent these kind of adverse events [36].

This study also showed a correlation between the risk of serious ADR and age classes. Children and infants had a significantly lower risk of serious ADRs compared to adolescents, as reported in other studies [37, 38]. To the best of our knowledge, no other publications have previously reported age as a risk factor for serious ADRrelated ED admission in children. In general, age-based analyses do not follow a clear pattern and are difficult to evaluate due to the variety of age classifications [10]. Healthcare professionals have to consider important factors related to age, such as pharmacokinetic and pharmacodynamic differences during children growth (i.e., immature tubular function and hypoalbuminemia in neonates, immaturity of blood brain barrier, reduced metabolism and liver function, etc.) [39].

Concerning risk factors associated with ADRs in children, polypharmacy was found as a potential predictor of adverse events. Multiple regression analysis showed a statistically significant correlation between polypharmacy (use of more than 3 drugs) and the risk of presenting to the ED for a serious ADR. Our results are consistent with several published investigations conducted in pediatric patients that also show polypharmacy to be an important factor that predisposes to ADRs [38]. Polypharmacy as a risk factor for ADRs is well characterized in adults and older subjects [40] and descriptive data are also available in children [41], but the risk of serious ADRs had not been addressed previously.

Off-label prescribing has been widely observed in children [42]. However, administration of a drug outside the conditions assessed during clinical trials may result in ADRs [43]. The present study shows no statistically significant difference between on-label and off-label use in terms of ADRs occurrence. Our evidence confirms the data already reported by Palmaro and colleagues on the safety of off-label drug utilization [44].

It is well known that the lack of reliable data on drug safety in the pediatric population is associated with specific issues, among which are the lack of dedicated clinical trials and the non-linear development of pharmacokinetic parameters [45]. Serious ADRs are generally not observed during pediatric clinical trials, especially in case of a latency period before onset [46], and in children as in adults it is not possible to fully investigate the spectrum of serious ADRs prior authorization of most medications [47]. Pharmacovigilance spontaneous reporting systems are subjected to under-reporting of ADRs, including serious ones [48]. Thus, an active pharmacovigilance study like the one presented here represents one of the best 
strategies to systematically collect, analyze and interpret data on ADRs [17], by means of protocols designed to actively detect ADRs on an ongoing basis within a defined group of people (i.e., children in ED).

The present study has several points of strength. First, we used a computerized monitoring programs and all participants were specifically trained healthcare professionals, who systematically analyzed each single ED clinical record. Furthermore, this is the first retrospective analysis evaluating serious ADRs as cause of ED admission in children over a long period of time in Italy. However, this study has also some limitations. The retrospective nature of our study may have led to an underestimation of the rate of ADR-related ED admissions as a result of missing documented clinical data. Indeed, not all pediatric ADRs were identified, since not all pediatric patients presenting an ADR, even if serious, attend ED. Therefore, the real prevalence of ADR is not known. Last, a control group with no ADRs was not available and this is also a limitation with regard to assessment of causality between ADRs and ED admission.

\section{Conclusions}

Serious ADRs are a relevant clinical event in children and a challenge for pediatricians and health care systems. The present research provided new insights on the factors that might increase the risk of serious pediatric ADRs. Future prospective, large-sample and multicenter studies should focus on other at-risk pediatric settings such as oncology, hematology, neurology, etc. to better understand the impact of ADRs and the effect of programmed preventive actions. In this context, we believe that active surveillance in pharmacovigilance might represent the best strategy to estimate and characterize the clinical burden of ADRs in children, with the final goal of improving the appropriateness of prescribing in the fragile population of pediatric patients.

\section{Additional files}

Additional file 1: Table S1. Most frequent suspect APs among antinfective for systemic use agents (ATC class J) and nervous system medications (ATC class N), overall and stratified according to ADR seriousness. This table reported the most frequent suspected active principles (APs) among antinfectives for systemic use agents and nervous system medications. (DOCX $48 \mathrm{~kb}$ )

Additional file 2: Table S2. Most frequent suspect vaccines, overall and stratified according to seriousness. This table reported the most frequent suspected vaccines. (DOCX $14 \mathrm{~kb}$ )

\section{Abbreviations}

ADR: Adverse Drug Reaction; AP: Active Principles; ATC: Anatomical Therapeutic Chemical; Cl: Confidence Interval; DDI: Drug-Drug Interactions; ED: Emergency Department; ROR: Reporting Odds Ratio; SOC: System Organ Class; WHO: World Health Organization

\section{Funding}

This study was funded by a research grant from the AIFA (the Italian Medicines Agency), Rome, Italy, Tuscan County resolution DGRT 790/2016 All. C. The funder of the study had no role in the collection, analysis and interpretation of data, nor in the writing of the report, nor in the decision to submit the article for publication.

\section{Availability of data and materials}

The datasets generated and/or analysed during the current study are not publicly available because they are property of the emergency department involved in the study, but are available from the corresponding author on reasonable request.

\section{Authors' contributions}

NL, GC, AM, LG, AV (Vannacci) made substantial contributions to conception and design of the study. EM, AV (Vitiello), RB, AMC, SM made substantial contributions to acquisition of data. NL, GC, AB, RB, EL, AV (Vannacci) made substantial contributions to analysis and interpretation of data. NL, GC, AB were involved in drafting the manuscript. EL, AM, LG, AV (Vannacci) were involved in revising the manuscript critically for important intellectual content. All authors gave final approval of the version to be published. All authors agreed to be accountable for all aspects of the work in ensuring that questions related to the accuracy or integrity of any part of the work are appropriately investigated and resolved. All authors read and approved the final manuscript.

\section{Ethics approval and consent to participate}

Tuscany (Italy) approved the present study with the Notification number 1225 - December 21, 2009. The Local Institutional Ethics Committee of the coordinating center (Comitato Etico di Area Vasta Nord "CEAVNO" per la Sperimentazione Clinica, Azienda Ospedaliero-Universitaria Pisana) approved the study according to the legal requirements concerning observational studies (Study number 3055/2010, Protocol number 45288 - August 6, 2014). For this type of study patient's consent to participate is not requested. In particular, the study protocol reported: "On the basis of the total amount of emergency department admissions per month, the commitment necessary for the collection of informed consent in Anna Meyer Children's University Hospital for the study period is logistically incompatible with the conduct of the present study", [...] "In the light of the specific activities of the emergency department, generally of urgent nature, the collection of informed consent is not compatible with the possibility of guaranteeing the conduct of normal clinical practice". The Local Institutional Ethics Committee of the coordinating center did not request patient's consent to participate to the present study.

\section{Competing interests}

The authors declare that they have no competing interests.

\section{Publisher's Note}

Springer Nature remains neutral with regard to jurisdictional claims in published maps and institutional affiliations.

\section{Author details}

'Department of Neurosciences, Psychology, Drug Research and Child Health, Section of Pharmacology and Toxicology, University of Florence, viale G. Pieraccini, 6, 50139 Florence, Italy. ${ }^{2}$ Department of Pharmacy, Anna Meyer Children's University Hospital, viale G. Pieraccini, 24, 50139 Florence, Italy. ${ }^{3}$ Department of Emergency Medicine, Anna Meyer Children's University

Hospital, viale G. Pieraccini, 24, 50139 Florence, Italy.

Received: 18 January 2018 Accepted: 8 April 2018

Published online: 16 April 2018

\section{References}

1. Shehab N, Lovegrove MC, Geller Al, Rose KO, Weidle NJ, Budnitz DS. US emergency department visits for outpatient adverse drug events, 20132014. JAMA. 2016;316(20):2115-25.

2. Budnitz DS, Lovegrove MC, Shehab N, Richards CL. Emergency hospitalizations for adverse drug events in older Americans. N Engl J Med. 2011;365(21):2002-12.

3. Pirmohamed M, James S, Meakin S, Green C, Scott AK, Walley TJ, Farrar K, Park BK, Breckenridge AM. Adverse drug reactions as cause of admission to hospital: prospective analysis of 18820 patients. BMJ. 2004;329(7456):15-9. 
4. Marengoni A, Pasina L, Concoreggi C, Martini G, Brognoli F, Nobili A, Onder G, Bettoni D. Understanding adverse drug reactions in older adults through drug-drug interactions. Eur J Intern Med. 2014;25(9):843-6.

5. Davies EA, O'Mahony MS. Adverse drug reactions in special populations the elderly. Br J Clin Pharmacol. 2015;80(4):796-807.

6. Alhawassi TM, Krass I, Bajorek BV, Pont LG. A systematic review of the prevalence and risk factors for adverse drug reactions in the elderly in the acute care setting. Clin Interv Aging. 2014;9:2079-86.

7. Salvi F, Marchetti A, D'Angelo F, Boemi M, Lattanzio F, Cherubini A. Adverse drug events as a cause of hospitalization in older adults. Drug Saf. 2012; 35(Suppl 1):29-45

8. Dehours E, Bounes V, Bagheri H, Valle B, Ducasse JL, Montastruc JL. Adverse drug reactions in an emergency medical dispatching Centre. Eur J Clin Pharmacol. 2014;70(7):881-7.

9. Samoy LJ, Zed PJ, Wilbur K, Balen RM, Abu-Laban RB, Roberts M. Drug-related hospitalizations in a tertiary care internal medicine service of a Canadian hospital: a prospective study. Pharmacotherapy. 2006;26(11):1578-86.

10. Smyth RM, Gargon E, Kirkham J, Cresswell L, Golder S, Smyth R, Williamson P. Adverse drug reactions in children-a systematic review. PLoS One. 2012 7(3):e24061.

11. Kearns GL, Abdel-Rahman SM, Alander SW, Blowey DL, Leeder JS, Kauffman RE. Developmental pharmacology-drug disposition, action, and therapy in infants and children. N Engl J Med. 2003;349(12):1157-67.

12. Mitchell AA, Lacouture PG, Sheehan JE, Kauffman RE, Shapiro S. Adverse drug reactions in children leading to hospital admission. Pediatrics. 1988; 82(1):24-9.

13. Turner S, Longworth A, Nunn AJ, Choonara I. Unlicensed and off label drug use in paediatric wards: prospective study. BMJ. 1998;316(7128):343-5.

14. Bates DW, Spell N, Cullen DJ, Burdick E, Laird N, Petersen LA, Small SD, Sweitzer BJ, Leape LL. The costs of adverse drug events in hospitalized patients. Adverse drug events prevention study group. JAMA. 1997;277(4): 307-11.

15. Zed PJ, Haughn C, Black KJ, Fitzpatrick EA, Ackroyd-Stolarz S, Murphy NG, MacKinnon NJ, Curran JA, Sinclair D. Medication-related emergency department visits and hospital admissions in pediatric patients: a qualitative systematic review. J Pediatr. 2013;163(2):477-83.

16. Carnovale C, Brusadelli T, Zuccotti G, Beretta S, Sullo MG, Capuano A, Rossi F, Moschini M, Mugelli A, Vannacci A, et al. The importance of monitoring adverse drug reactions in pediatric patients: the results of a national surveillance program in Italy. Expert Opin Drug Saf. 2014;13(Suppl 1):S1-8.

17. Perrone V, Conti V, Venegoni M, Scotto S, Degli Esposti L, Sangiorgi D, Prestini L, Radice S, Clementi E, Vighi G. Seriousness, preventability, and burden impact of reported adverse drug reactions in Lombardy emergency departments: a retrospective 2-year characterization. Clinicoecon Outcomes Res. 2014;6:505-14.

18. Naranjo CA, Busto U, Sellers EM, Sandor P, Ruiz I, Roberts EA, Janecek E, Domecq C, Greenblatt DJ. A method for estimating the probability of adverse drug reactions. Clin Pharmacol Ther. 1981;30(2):239-45.

19. Schumock GT, Thornton JP. Focusing on the preventability of adverse drug reactions. Hosp Pharm. 1992;27(6):538.

20. Causality assessment of adverse event following immunization (AEFI): user manual for the revised WHO. classification. Available at: http://www.who.int/ vaccine_safety/publications/aevi_manual.pdf [Last Accessed 13 Oct 2017].

21. Tozzi AE, Asturias EJ, Balakrishnan MR, Halsey NA, Law B, Zuber PL. Assessment of causality of individual adverse events following immunization (AEFI): a WHO tool for global use. Vaccine. 2013;31(44):5041-6.

22. Micromedex ${ }^{\circledast}$ Medication, Disease and Toxicology Management. Available at: http://www.micromedexsolutions.com/home/dispatch.

23. Santos TRA, Silveira EA, Pereira LV, Provin MP, Lima DM, Amaral RG. Potential drug-drug interactions in older adults: a population-based study. Geriatr Gerontol Int. 2017;17(12):2336-46.

24. Neubert A, Wong IC, Bonifazi A, Catapano M, Felisi M, Baiardi P, Giaquinto C, Knibbe CA, Sturkenboom MC, Ghaleb MA, et al. Defining off-label and unlicensed use of medicines for children: results of a Delphi survey. Pharmacol Res. 2008;58(5-6):316-22

25. Rosafio C, Paioli S, Del Giovane C, Cenciarelli V, Viani N, Bertolani P, lughetti L. Medication-related visits in a pediatric emergency department: an 8-years retrospective analysis. Ital J Pediatr. 2017;43(1):55.

26. Ferrajolo C, Capuano A, Trifiro G, Moretti U, Rossi F, Santuccio C. Pediatric drug safety surveillance in Italian pharmacovigilance network: an overview of adverse drug reactions in the years 2001 - 2012. Expert Opin Drug Saf. 2014;13(Suppl 1):S9-20.

27. Lusini G, Lapi F, Sara B, Vannacci A, Mugelli A, Kragstrup J, Bjerrum L. Antibiotic prescribing in paediatric populations: a comparison between Viareggio, Italy and Funen, Denmark. Eur J Pub Health. 2009;19(4):434-8.

28. Priyadharsini R, Surendiran A, Adithan C, Sreenivasan S, Sahoo FK. A study of adverse drug reactions in pediatric patients. J Pharmacol Pharmacother. 2011;2(4):277-80.

29. Signorelli C, Chiesa V, Odone A. Meningococcal serogroup B vaccine in Italy: state-of-art, organizational aspects and perspectives. J Prev Med Hyg. 2015; 56(3):E125-32.

30. The Medicines Utilization Monitoring Centre. National Report on medicines use in Italy 2016. Rome: Italian Medicines Agency; 2017. http://www.aifa.gov.it/sites/ default/files/Rapporto_OsMed_2016_AIFA.pdf [Last Accessed 13 Oct 2017

31. Staltari O, Cilurzo F, Caroleo B, Greco A, Corasaniti F, Genovesi MA, Gallelli L. Annual report on adverse events related with vaccines use in Calabria (Italy): 2012. J Pharmacol Pharmacother. 2013:4(Suppl 1):S61-5.

32. Lavonas EJ, Banner W, Bradt P, Bucher-Bartelson B, Brown KR, Rajan P, Murrelle L, Dart RC, Green JL. Root causes, clinical effects, and outcomes of unintentional exposures to buprenorphine by young children. J Pediatr. 2013;163(5):1377-83. e1371-1373

33. Schillie SF, Shehab N, Thomas KE, Budnitz DS. Medication overdoses leading to emergency department visits among children. Am J Prev Med. 2009; 37(3):181-7.

34. Franklin RL, Rodgers GB. Unintentional child poisonings treated in United States hospital emergency departments: national estimates of incident cases, population-based poisoning rates, and product involvement. Pediatrics. 2008:122(6):1244-51.

35. Wiley CC, Wiley JF 2nd. Pediatric benzodiazepine ingestion resulting in hospitalization. J Toxicol Clin Toxicol. 1998;36(3):227-31.

36. Kaushal R, Bates DW, Landrigan C, McKenna KJ, Clapp MD, Federico F, Goldmann DA. Medication errors and adverse drug events in pediatric inpatients. JAMA. 2001:285(16):2114-20.

37. Knopf H, Du Y. Perceived adverse drug reactions among non-institutionalized children and adolescents in Germany. Br J Clin Pharmacol. 2010;70(3):409-17.

38. Impicciatore $P$, Choonara I, Clarkson A, Provasi D, Pandolfini C, Bonati M. Incidence of adverse drug reactions in paediatric in/out-patients: a systematic review and meta-analysis of prospective studies. Br J Clin Pharmacol. 2001; 52(1):77-83.

39. Anderson GD, Lynn AM. Optimizing pediatric dosing: a developmental pharmacologic approach. Pharmacotherapy. 2009;29(6):680-90.

40. Nobili A, Licata G, Salerno F, Pasina L, Tettamanti M, Franchi C, De Vittorio L, Marengoni A, Corrao S, lorio A, et al. Polypharmacy, length of hospital stay, and in-hospital mortality among elderly patients in internal medicine wards. The REPOSI study. Eur J Clin Pharmacol. 2011;67(5):507-19.

41. Morden NE, Goodman D. Pediatric polypharmacy: time to lock the medicine cabinet? Arch Pediatr Adolesc Med. 2012;166(1):91-2.

42. Lindell-Osuagwu L, Hakkarainen M, Sepponen K, Vainio K, Naaranlahti T, Kokki H. Prescribing for off-label use and unauthorized medicines in three paediatric wards in Finland, the status before and after the European Union Paediatric regulation. J Clin Pharm Ther. 2014;39(2):144-53.

43. Cuzzolin L, Atzei A, Fanos V. Off-label and unlicensed prescribing for newborns and children in different settings: a review of the literature and a consideration about drug safety. Expert Opin Drug Saf. 2006;5(5):703-18.

44. Palmaro A, Bissuel R, Renaud N, Durrieu G, Escourrou B, Oustric S, Montastruc $J$, Lapeyre-Mestre M. Off-label prescribing in pediatric outpatients. Pediatrics. 2015:135(1):49-58

45. Napoleone E. Children and ADRs (adverse drug reactions). Ital J Pediatr. 2010;36:4

46. Clavenna A, Berti A, Gualandi L, Rossi E, De Rosa M, Bonati M. Drug utilisation profile in the Italian paediatric population. Eur J Pediatr. 2009. 168(2):173-80.

47. Clavenna A, Bonati M. Adverse drug reactions in childhood: a review of prospective studies and safety alerts. Arch Dis Child. 2009;94(9):724-8.

48. Langerova P, Vrtal J, Urbanek K. Adverse drug reactions causing hospital admissions in childhood: a prospective, observational, single-Centre study. Basic Clin Pharmacol Toxicol. 2014;115(6):560-4. 